\title{
LIE CENTRAL TRIPLE RACKS
}

\author{
Guy Roger Biyogmam
}

Received: 18 February 2014; Revised 2 September 2014

Communicated by A. Çiğdem Özcan

\begin{abstract}
This paper introduces Lie $\mathfrak{c}$-triple racks. These triple systems generalize both left and right racks to ternary algebras, and locally differentiates to $g b$-triple systems [3].
\end{abstract}

Mathematics Subject Classification (2010): 05B07, 18E10, 17A99

Keywords: $g b$-Triple systems, Lie triple systems, Lie racks

\section{Introduction}

The generalization of Lie algebras to algebras such as Lie triple systems, Jordan triple systems [8] and 3-Lie algebras [7] suggests a natural generalization of Leibniz algebras (non commutative Lie algebras) [11] to ternary algebras. One generalization is provided by Leibniz 3-algebras [5] for which the characteristic identity expresses the adjoint action as a derivation of the algebra. A second generalization of Leibniz algebras to ternary algebras is provided by Leibniz triple systems [4]. They are defined in such a way that Lie triple systems are a particular case. Recently, the author introduced $g b$-triple systems [3], another generalization of Leibniz algebras in which the ternary operation $T: \mathfrak{g}^{\otimes 3} \rightarrow \mathfrak{g}$ expresses the map $T_{a, b}(x)=T(a, x, b)$ as a derivation of $\mathfrak{g}$ for all $a, b \in \mathfrak{g}$.

The local integration problem of these algebras generated from Lie's third theorem, which states that every finite-dimensional Lie algebra over the real numbers is associated with a Lie group. Partial solutions to this problem for Leibniz algebras (dubbed by Loday as the Coquecigrue problem) have been provided by several authors (see M. Kinyon [10], S. Covez [6]). The author extended Kinyon's results to Leibniz 3-algebras using Lie 3-racks [2]. In this paper we open the problem of integration of $g b$-triple systems. We follow Kinyon's approach [10] to open a path to a solution by defining an algebraic structure that locally differentiates to a $g b$-triple systems. We refer to these algebras as Lie c-triple racks. They appear to generalize both left and right Lie racks to ternary algebras; a particularity not supported by Lie 3-racks. 
For the remainder of this paper, we assume that $\mathfrak{K}$ is a field of characteristic different to 2 .

\section{2. c-Triple racks}

In this section we define $\mathfrak{c}$-triple racks and provide some examples. We also provide funtorial connections with the category of groups and the category of racks.

Recall that a $g b$-triple system [3] is a $\mathfrak{K}$-vector space $\mathfrak{g}$ equipped with a trilinear operation $[-,-,-]_{\mathfrak{g}}: \mathfrak{g}^{\otimes 3} \longrightarrow \mathfrak{g}$ satisfying the identity

$\left[A, B,[X, C, Y]_{\mathfrak{g}}\right]_{\mathfrak{g}}=\left[X,[A, B, C]_{\mathfrak{g}}, Y\right]_{\mathfrak{g}}-\left[[X, A, Y]_{\mathfrak{g}}, B, C\right]_{\mathfrak{g}}-\left[A,[X, B, Y]_{\mathfrak{g}}, C\right]_{\mathfrak{g}}(1)$

Definition 2.1. A c-triple rack $\left(R,[-,-,-]_{R}\right)$ is a set $R$ together with a ternary operation $[-,-,-]_{R}: R \times R \times R \longrightarrow R$ satisfying the following conditions:

(1) $\left[x,[a, b, c]_{R}, y\right]_{R}=\left[[x, a, y]_{R},[x, b, y]_{R},[x, c, y]_{R}\right]_{R}$ (c-distributive property),

(2) Given $a, c, d \in R$, there exists a unique $x \in R$ such that $[a, x, c,]_{R}=d$.

Definition 2.2. A c-triple rack $\left(R,[-,-,-]_{R}\right)$ is said to be pointed if there is a distinguished element $1 \in R$ satisfying

$$
[1, y, 1]_{R}=y \text { and }[a, 1, b]_{R}=1 \text { for all } a, b \in R .
$$

A $\mathfrak{c}$-triple rack is said to be a weak $\mathfrak{c}$-triple quandle if it satisfies

$$
[x, x, x]_{R}=x \text { for all } x \in R .
$$

A $\mathfrak{c}$-triple rack is a $\mathfrak{c}$-triple quandle if it satisfies

$$
[a, y, b]_{R}=y \text { if } a=y \text { or } b=y .
$$

Note that these generalize the notions of racks and quandles [9] to ternary operations. It is also clear that $\mathfrak{c}$-triple quandles are weak $\mathfrak{c}$-triple quandles but the converse is not true. See Example 2.4.

Definition 2.3. Let $R$ and $R^{\prime}$ be two c-triple racks. A function $\alpha: R \longrightarrow R^{\prime}$ is said to be a homomorphism of $\mathfrak{c}$-triple racks if

$$
\alpha\left([x, y, z]_{R}\right)=[\alpha(x), \alpha(y), \alpha(z)]_{R^{\prime}} \text { for all } x, y, z \in R .
$$

This provides a category ${ }_{c} p R A C K$ of pointed $\mathfrak{c}$-triple racks and pointed $\mathfrak{c}$-triple rack homomorphisms.

Example 2.4. Let $\Gamma:=\mathbf{Z}\left[t^{ \pm 1}, s\right] /\left(2 s^{2}+t s-s\right)$. Any $\Gamma$-module $M$ together with the ternary operation $[-,-,-]_{M}$ defined by

$$
[a, b, c]_{M}=s a+t b+s c
$$


is a c-triple rack. Indeed,

$$
\begin{aligned}
{\left[[x, a, y]_{R},[x, b, y]_{R},[x, c, y]_{R}\right]_{R} } & =s(s x+t a+s y)+t(s x+t b+s y)+s(s x+t c+s y) \\
& =\left(2 s^{2}+s t\right)(x+y)+t s(a+c)+t^{2} b \\
& =\left[x,[a, b, c]_{R}, y\right]_{R} \text { since } 2 s^{2}+s t=s .
\end{aligned}
$$

Therefore the $\mathfrak{c}$-distributive property is satisfied. For the second axiom, given a, $c, d \in$ $R$ one checks that $x:=t^{-1}(d-s(a+c))$ uniquely satisfies $[a, x, c]_{M}=d$. Note that $M$ is a weak $\mathfrak{c}$-triple quandle that is not a $\mathfrak{c}$-triple quandle.

Example 2.5. Let $G$ be a group with identity 1, and define on $G$ the operation $[-,-,-]_{G}$ by

$$
[a, b, c]_{G}=a c b c^{-1} a^{-1} .
$$

Then $(G,[-,-,-], 1)$ is a pointed weak $\mathfrak{c}$-triple quandle. Indeed, we have on one hand

$$
\left[x,[a, b, c]_{G}, y\right]_{G}=x y[a, b, c]_{G} y^{-1} x^{-1}=x y a c b^{-1} c^{-1} a^{-1} y^{-1} x^{-1} .
$$

On the other hand,

$$
\begin{gathered}
{\left[[x, a, y]_{G},[x, b, y]_{G},[x, c, y]_{G}\right]_{G}=\left[x y a y^{-1} x^{-1}, x y b y^{-1} x^{-1}, x y c y^{-1} x^{-1}\right]_{G}} \\
=x y a y^{-1} x^{-1} x y c y^{-1} x^{-1} x y b^{-1} y^{-1} x^{-1} x y c^{-1} y^{-1} x^{-1} x y a^{-1} y^{-1} x^{-1} \\
=x y a c b^{-1} c^{-1} a^{-1} y^{-1} x^{-1} \text { by cancellation. }
\end{gathered}
$$

Therefore the $\mathfrak{c}$-distributive property is satisfied. For the second axiom, given $a, c, d \in G$ one checks that $x:=c^{-1} a^{-1} d a c$ uniquely satisfies $[a, x, c]_{M}=d$. Finally, it is clear that $[x, x, x]_{R}=x$ for all $x \in R$.

As a consequence, we have the following:

Proposition 2.6. There is a faithful functor $\mathfrak{F}$ from the category of groups to the category of pointed $\mathfrak{c}$-triple racks.

Proof. Define $\mathfrak{F}$ by $\mathfrak{F}(G)=(G,[-,-,-], 1)$ as in Example 2.5. Its left adjoint $\mathfrak{F}^{\prime}$ is defined as follows: Given a pointed $\mathfrak{c}$-triple rack $R$, consider the quotient group

$$
G_{R}=<R>/ I
$$

where $\langle R>$ is the free group on $R$ and $I$ is the normal subgroup of $<R>$ generated by the set $\left\{\left(a^{-1} c^{-1} b^{-1} c a\right)\left([a, b, c]_{R}\right)\right.$ : with $\left.a, b, c \in R\right\}$. Indeed, given a morphism of $\mathfrak{c}$-triple racks $\alpha: R \longrightarrow \mathfrak{F}(G)$, there is a unique morphism of groups $\beta:<R>\longrightarrow G$ such that $\alpha=\left.\beta\right|_{R}$ by the universal property of free groups. So $\beta\left(\left(a^{-1} c^{-1} b^{-1} c a\right)\left([a, b, c]_{R}\right)\right)=\alpha\left(\left(a^{-1} c^{-1} b^{-1} c a\right)\left([a, b, c]_{R}\right)\right)=1$ for all $a, b, c \in R$. 
Now by the universal property of quotient groups, there is a unique morphism of groups $\alpha_{*}: \mathfrak{F}^{\prime}(R) \longrightarrow G$ such that the following diagram commutes.

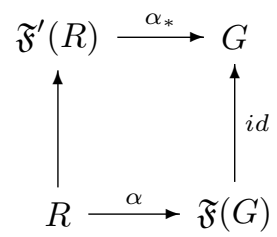

Example 2.7. Let $(R, \circ, 1)$ be a pointed rack. Then define on $R$ the ternary operation by

$$
[a, b, c]_{R}=a \circ(c \circ b)
$$

It is easy to show that $(R,[-,-,-], 1)$ is a pointed $\mathfrak{c}$-triple rack.

As a consequence we have the following:

Proposition 2.8. There is a faithful functor $\mathfrak{H}$ from the category of pointed racks to the category of $\mathrm{c}$-triple racks.

Proof. Define $\mathfrak{H}$ by $\mathfrak{H}((R, \circ, 1))=(R,[-,-,-], 1)$ as in Example 2.7. Now, given a pointed $\mathfrak{c}$-triple rack $(R,[-,-,-], 1)$, one easily checks that the set $R^{\times(3)}$ together with the binary operation

$$
(a, b, c) \circ(x, y, z)=\left([a, x, c]_{R},[a, y, c]_{R},[a, z, c]_{R}\right)
$$

is a rack pointed at $(1,1,1)$. We then define the left adjoint $\mathfrak{H}^{\prime}$ of $\mathfrak{H}$ by

$$
\mathfrak{H}^{\prime}((R,[-,-,-], 1))=\left(R^{\times(3)}, \circ,(1,1,1)\right) .
$$

Let us observe that in the proof of Proposition 2.8 the set $R^{\times(3)}$ is a quandle if $R$ is a c-triple quandle.

\section{From Lie c-triple racks to gb-triple systems}

In this section we define the notion of Lie $\mathfrak{c}$-triple racks. We show that the tangent functor $T_{1}$ locally (at a specific point) maps Lie $\mathfrak{c}$-triple racks to gb-triple system.

Definition 3.1. A pointed $\mathfrak{c}$-triple $\operatorname{rack}\left(R,[-,-,-]_{R}, 1\right)$ is called a Lie $\mathfrak{c}$-triple rack if the underlying set $R$ is a differentiable manifold and the ternary operation $[-,-,-]_{R}: R \times R \times R \longrightarrow R$ is a smooth mapping.

Note that this definition appears to extend both left and right Lie racks [1] to ternary operations. 
Example 3.2. Let $G$ be a Lie group endowed with the operation

$$
[a, b, c]_{G}=a c b c^{-1} a^{-1} .
$$

It follows by Example 2.5 that $G$ is a Lie $\mathfrak{c}$-triple rack.

Example 3.3. Let $(H,\{-,-,-\})$ be a group endowed with an antisymmetric ternary operation and $V$ an $H$-module. Define the ternary operation $[-,-,-]_{R}$ on $R:=$ $V \times H$ by

$$
[(a, A),(b, B),(c, C)]_{R}:=\left(\{A, B, C\} b, A C B C^{-1} A^{-1}\right),
$$

where $a, b, c \in V$ and $A, B, C \in H$. Then $\left(R,[-,-,-]_{R},(0,1)\right)$ is a Lie c-triple rack.

For a pointed $\mathfrak{c}$-triple rack $R$, consider

$$
\operatorname{Aut}(R):=\left\{\xi: R \rightarrow R, \xi \text { smooth bijection }: \xi\left([a, b, c]_{R}\right)=[\xi(a), \xi(b), \xi(c)]_{R}\right\}
$$

and let $\phi: R \times R \times R \rightarrow R$ be the mapping given by $\phi(a, b, c)=[a, b, c]_{R}$. We have as a consequence of the second axiom of Definition 2.1 that the map $D: R \times$ $R \longrightarrow \operatorname{Aut}(R),(a, c) \mapsto D(a, c)=\phi_{(a, c)}$ where $\phi_{(a, c)}(x)=[a, x, c]_{R}$ for all $x \in R$, is well-defined differentiable map. Let $D_{*}: \mathfrak{g} \times \mathfrak{g} \longrightarrow g l(\mathfrak{g})$ be the induced map of tangent spaces, where $\mathfrak{g}:=T_{1} R$ is the tangent space of $R$ at the point 1 and $g l(\mathfrak{g})$ is the Lie algebra associated to $G L(\mathfrak{g})$. Define a trilinear bracket on $\mathfrak{g}$ by

$$
[X, Y, Z]_{\mathfrak{g}}=D_{*}(X, Z)(Y) .
$$

Proposition 3.4. Let $\left(R,[-,-,-]_{R}, 1\right)$ be a pointed $\mathfrak{c}$-triple rack. Then $D(a, c) \in$ $\operatorname{Aut}(R)$ for all $a, c \in R$.

\section{Proof.}

$$
\begin{aligned}
D(a, c)\left([x, y, x]_{R}\right) & =\phi(a, c)\left([x, y, z]_{R}\right) \\
& =\left[a,[x, y, z]_{R}, c\right]_{R} \\
& =\left[[a, x, c]_{R},[a, y, c]_{R},[a, z, c]_{R}\right]_{R} \text { by Definition } 2.1 \\
& =\left[\phi_{(a, c)}(x), \phi_{(a, c)}(y), \phi_{(a, c)}(z)\right]_{R} \\
& =[D(a, c)(x), D(a, c)(y), D(a, c)(z)]_{R} .
\end{aligned}
$$

Remark 3.5. Note that for all $a, c \in R, R$ acts on itself (considered as a differentiable manifold) via the maps $\phi_{(a, c)}$ by Proposition 3.4. Also, $\phi_{(a, c)}(1)=$ $[a, 1, c]_{R}=1$. So the tangent functor $T_{1}$ applied to $\phi_{(a, c)}: R \longrightarrow R$ yields a linear map $\phi_{(a, c) *}: T_{1} R \longrightarrow T_{1} R$. Since $\phi(a, c) \in \operatorname{Aut}(R)$ by Proposition 3.4, it follows that $\phi_{(a, c) *} \in G L\left(T_{1} R\right)$. Now let $X \in T_{1} R$ and denote by $X_{1}:=\phi_{(a, c) *}(X)$ the vector field extension of $X$. Then $X_{1}$ is generated by a one-parameter family of 
diffeomorphisms $\gamma_{X}: \mathbb{R} \rightarrow R$ with initial point $\gamma_{X}(0)=1$ and initial tangent vector $d \gamma_{X}(0)=X$. The corresponding exponential map (see [12, Chapter 9]) denoted $\exp _{1}: T_{1}(R) \rightarrow R$ is then defined by $\exp _{1}(X)=\gamma_{X}(1)$.

Theorem 3.6. Let $\left(R,[-,-,-]_{R}, 1\right)$ be a Lie $\mathfrak{c}$-triple rack and $\mathfrak{g}:=T_{1} R$. Then for all $a, c \in R$, the tangent mapping $\phi_{(a, c) *}=T_{1}\left(\phi_{(a, c)}\right)$ is an automorphism of $\mathfrak{g}$.

Proof. Let $X, Y, Z \in \mathfrak{g}$ and let $x, y, z$ be respectively the images of $X, Y$ and $Z$ by the exponential map $\exp _{1}$ (see Remark 3.5). By the $\mathfrak{c}$-distributive property of c-triple racks, we have

$$
\phi_{(a, c)}\left(\phi_{(x, z)}(y)\right)=\phi_{\left(\phi_{(a, c)}(x), \phi_{(a, c)}(z)\right)}\left(\phi_{(a, c)}(y)\right)
$$

which when successively differentiated at $1 \in R$ with respect to the parameter $\gamma_{Y}$ then $\gamma_{Z}$ then $\gamma_{X}$ yields

$$
\phi_{(a, c) *}\left([X, Y, Z]_{\mathfrak{g}}\right)=\left[\phi_{(a, c) *}(X), \phi_{(a, c) *}(Y), \phi_{(a, c) *}(Z)\right]_{\mathfrak{g}}
$$

Theorem 3.7. Let $R$ be a Lie $\mathfrak{c}$-triple rack and $A, C \in \mathfrak{g}:=T_{1} R$. Let a,c be

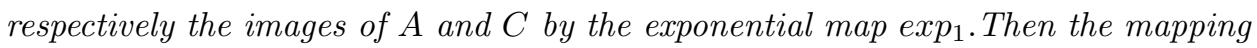
$D_{(A, C) *}: \mathfrak{g} \longrightarrow g l(\mathfrak{g})$ is a derivation of $\mathfrak{g}$. Moreover, $D_{(A, C) *}$ is exactly $T_{1}(\Phi)$, where $\Phi$ is the mapping $\Phi: R \times R \longrightarrow G L(\mathfrak{g})$ defined by $\Phi(a, c)=\phi_{(a, c) *}$.

Proof. From the proof of Theorem 3.6, $\phi_{(a, c) *} \in G L(\mathfrak{g})$. In addition, we have $\phi_{(1,1) *}=I$, where $I$ is the identity of $G L(\mathfrak{g})$. Now differentiating $\Phi$ at $(1,1)$ gives a map $T_{(1,1)}(\Phi): T_{1}(R \times R) \longrightarrow g l(\mathfrak{g})$. Also differentiating the identity $(2)$ above at $(1,1)$ with respect to $\gamma_{(A, C)}$ yields

$$
\begin{aligned}
D_{(A, C) *}\left(D_{(X, Z) *}(Y)\right)= & {\left[A,[X, Y, Z]_{\mathfrak{g}}, C\right]_{\mathfrak{g}} } \\
= & {\left[[A, X, C]_{\mathfrak{g}}, Y, Z\right]_{\mathfrak{g}}+\left[X,[A, Y, C]_{\mathfrak{g}}, Z\right]_{\mathfrak{g}}+\left[X, Y[A, Z, C]_{\mathfrak{g}}\right]_{\mathfrak{g}} } \\
= & D_{\left(D_{(A, C) *}(X), Z\right) *}(Y)+D_{(X, Z) *}\left(D_{(A, C) *}(Y)\right) \\
& +D_{\left(X, D_{(A, C) *}(Z)\right) *}(Y) .
\end{aligned}
$$

Hence $D_{(A, C) *}$ is a derivation of $\mathfrak{g}$ and the map $T_{1}(\Phi)$ is exactly $D_{(A, C) *}$.

From the calculations performed in the proofs of Theorem 3.6 and Theorem 3.7, we deduce that the ternary operation $[-,-,-]_{\mathfrak{g}}$ satisfies the identity $(1)$. We then have the following result:

Corollary 3.8. Let $R$ be a Lie $\mathfrak{c}$-triple rack and $\mathfrak{g}:=T_{1} R$. Then there exists a trilinear map $[-,-,-]_{\mathfrak{g}}: \mathfrak{g} \times \mathfrak{g} \times \mathfrak{g} \longrightarrow \mathfrak{g}$ such that $\left(\mathfrak{g},[-,-,-]_{\mathfrak{g}}\right)$ is a gb-triple system. 
Remark 3.9. Let $G$ be the Lie $\mathfrak{c}$-triple rack of Example 3.2 and $l$ the Lie algebra associated to the underlying group $G$. Then the bracket of the gb-triple system $\mathfrak{g}=$ $T_{1}(R)$ can be written in terms of the bracket of the Lie algebra $l$ as

$$
[X, Y, Z]_{\mathfrak{g}}=\left[Y,[X, Z]_{l}\right]_{l} .
$$

To check that $[-,-,-]_{\mathfrak{g}}$ satisfies the identity (1), let $X, Y, A, B, C \in \mathfrak{g}$; we have on one hand

$$
\begin{aligned}
{\left[X, Y,[A, B, C]_{\mathfrak{g}}\right]_{\mathfrak{g}}+\left[[A, X, C]_{\mathfrak{g}}, Y, B\right]_{\mathfrak{g}} } & =\left[Y,\left[X,[A, B, C]_{\mathfrak{g}}\right]_{l}\right]_{l}+\left[Y,\left[[A, X, C]_{\mathfrak{g}}, B\right]_{l}\right]_{l} \\
& =\left[Y,\left[X,\left[B,[A, C]_{l}\right]_{l}\right]_{l}+\left[\left[X,[A, C]_{l}\right]_{l}, B\right]_{l}\right]_{l} \\
& =\left[Y,\left[[X, B]_{l},[A, C]_{l}\right]_{l}\right]_{l} .
\end{aligned}
$$

On the other hand,

$$
\begin{aligned}
{\left[A,[X, Y, B]_{\mathfrak{g}}, C\right]_{\mathfrak{g}}-\left[X,[A, Y, C]_{\mathfrak{g}}, B\right]_{\mathfrak{g}} } & =\left[[X, Y, B]_{\mathfrak{g}},[A, C]_{l}\right]_{l}-\left[[A, Y, C]_{\mathfrak{g}},[X, B]_{l}\right]_{l} \\
& =\left[\left[Y,[X, B]_{l}\right]_{l},[A, C]_{l}\right]_{l}-\left[\left[Y,[A, C]_{l}\right]_{l},[X, B]_{l}\right]_{l} .
\end{aligned}
$$

The equality holds by the Jacoby identity.

Acknowledgment. The author would like to thank the referee for very helpful comments and suggestions.

\section{References}

[1] N. Andruskiewitsch and M. Graña, From racks to pointed Hopf algebras, Adv. Math., 178(2) (2003), 177-243.

[2] G. R. Biyogmam, Lie n-rack, C. R. Math. Acad. Sci. Paris, 349(17-18) (2011), 957-960.

[3] G. R. Biyogmam, Introduction to gb-triple systems, ISRN Algebra 2014, Art. ID 738154, 5 pp.

[4] M. R. Bremner and J. Sánchez-Ortega, Leibniz triple systems, Commun. Contemp. Math., 16(1) (2014), 1350051, 19 pp.

[5] J. M. Casas, J.-L. Loday and T. Pirashvili, Leibniz n-algebras, Forum Math., 14(2) (2002), 189-207.

[6] S. Covez, The local integration of Leibniz algebras, Ann. Inst. Fourier (Grenoble), 63(1) (2013), 1-35.

[7] V. T. Filippov, n-Lie algebras, Sibirsk. Mat. Zh., 26(6) (1985), 126-140.

[8] N. Jacobson, Lie and Jordan triple systems, Amer. J. Math., 71 (1949), 149170.

[9] D. Joyce, A classifying invariant of knots, the knot quandle, J. Pure Appl. Algebra, 23(1) (1982), 37-65. 
[10] M. K. Kinyon, Leibniz algebras, Lie racks and digroups, J. Lie Theory, 17(1) (2007), 99-114.

[11] J.-L. Loday, Une version non commutative des algebres de Lie: les algebres de Leibniz, Enseign. Math., 39 (1993), 269-293.

[12] M. Spivak, A Comprehensive Introduction to Differential Geometry, Vol. I, (2nd edition) Publish or Perish, Inc., Wilmington, Del., 1979.

\section{Guy Roger Biyogmam}

Department of Mathematics

Southwestern Oklahoma State University

100 Campus Drive

Weatherford, OK 73096, USA

e-mail: guy.biyogmam@swosu.edu 